\title{
Different Apolipoproteins Impact Nanolipoprotein Particle Formation
}

Brett A. Chromy, Erin Arroyo, Craig D. Blanchette, Graham Bench, Henry Benner, Jenny A. Cappuccio, Matthew A. Coleman, Paul T. Henderson, Angie K. Hinz, Edward A. Kuhn, Joseph B. Pesavento, Brent W. Segelke, Todd A. Sulchek, Ted Tarasow, Vicki L. Walsworth, and Paul D. Hoeprich*

Chemistry, Materials, and Life Sciences; Lawrence Livermore National Laboratory; Livermore, CA 94551

\section{Supporting Information}

\section{Experimental Section:}

Materials: Phospholipids (DMPC and NBD-DMPC) were purchased from Avanti Polar Lipids. Inc (Alabaster, AL). Full-length apoA-I was purchased from Fitzgerald, Inc. (Concord, MA), apoA-I, $\Delta$ 1-55 (MSP1T2) and Nanodisc ${ }^{\mathrm{TM}}$ particles were purchased from Nanodisc, Inc., (Urbana, IL). The latter particles were made from DMPC and apoAI, $\Delta$ 1-55 protein fragment (MSP1T2); this fragment has a modified $\mathrm{NH}_{2}$-terminus containing a His tag and a tobacco etch virus (TEV) cleavage site.

apoE422K and apoLp-III protein production: The expression clone to produce apoE422K, the N-terminal $22 \mathrm{kDa}$ fragment of apolipoprotein $\mathrm{E} 4$ (apoE4), as a 6His and thyrodoxin tagged construct was kindly provided by Dr. Karl Weisgraber. ApoE422K was over-expressed and in E. coli as previously reported ${ }^{1}$. Pelletted E. coli cells expressing apoE422K were re-suspended in lysis buffer (50 mM sodium phosphate, 300 $\mathrm{mM}$ sodium chloride, $10 \mathrm{mM}$ imidazole, $\mathrm{pH}$ 8.0) and lysed with an Emulsiflex-05 homogenizer (Avestin Inc., Ottawa, Canada) at $4^{\circ} \mathrm{C}$. Following centrifugation, the clarified supernatant was first partially purified by nickel affinity chromatography using a $5 \mathrm{ml}$ His Trap FF crude nickel column (GE Healthcare) on an Akta FPLC (GE 
Healthcare) then further purified with a $320 \mathrm{ml}$ Superdex 75 HiLoad 26/60 column (GE Healthcare) using TBS running buffer $(10 \mathrm{mM}$ Tris, $\mathrm{pH}$ 7.4, $0.15 \mathrm{M}$ sodium chloride, $0.25 \mathrm{mM}$ EDTA, $0.005 \%$ sodium azide) giving one predominant peak. The collected material was cleaved with bovine $\alpha$-Thrombin (Haematologic Technologies) 1/500 enzyme/protein for 1 hour at $37^{\circ} \mathrm{C}$. Resulting products were separated by SEC on a 320 ml Superdex 75 HiLoad 26/60 FPLC column with one column volume of TBS. Protein fractions were analyzed by SDS-PAGE gels stained with Sypro Ruby (BioRad), gels were imaged with a Typhooon 9410 (GE Healthcare). Relative purity of the proteins was determined to be greater than $95 \%$ by densitometry and overall yields are on the order of $6 \mathrm{mg} / \mathrm{L}$ bacterial culture.

The B. mori apoLp-III expression clone was a kind gift from Dr. Rob Ryan. ApoLp-III was over-expressed in E. coli as described ${ }^{2,3}$. The protein was expressed with a PEL leader sequence, targeting the protein to the periplasm, where the leader sequence is cleaved and protein secreted in to the media. Expression was induced for four hours, bacteria were pelleted and the supernatant was collected, filtered $(0.8 \mu \mathrm{m})$, and subsequently concentrated to a volume of $\sim 20 \mathrm{ml}$ using a Vivaflow 200 (Sartorius) with a 5-kDa MW cutoff PES membrane. The concentrated protein was exchanged against 20 mM Tris $\mathrm{pH} 8.0$ over a HiPrep 26/10 desalting column on an Akta FPLC (GE Healthcare). The protein was then purified to homogeneity by HPLC (Shimadzu) using a ProPac WAX-10 column (Dionex) and eluted as follows: 0-100\% gradient between $20 \mathrm{mM}$ Tris $\mathrm{pH} 8.0$ and $20 \mathrm{mM}$ Tris $\mathrm{pH} 8.0$ with $0.5 \mathrm{M} \mathrm{NaCl}$. Fractions showing highest protein content by $\mathrm{A}_{280}$ were pooled. Protein purity was checked by sodium 
dodecylsulfate-polyacrylamide gel electrophoresis (SDS-PAGE) and mass spectroscopy analysis. The protein was $>90 \%$ pure by gel electrophoresis, MS analysis gave expected molecular ion peaks; overall yield was $40 \%$.

Fluorescently labeled apoE422K was obtained by using a Cy3 labeling kit and following the manufacturer's instructions (GE Healthcare). Dye:protein ratio was determined by comparing the absorbance of the protein at $280 \mathrm{~nm}$ and the absorbance of the CyDye at $532 \mathrm{~nm}$. The ratio provided a 1:1 correlation suggesting that a single Cy3 molecule is present on each apoE422K protein.

Nanolipoprotein particle (NLP) formation: SI figure 1 schematically shows the assembly process while SI Table 1 details the NLP preparations undertaken in this study. DMPC (20 mg) is weighed out, added to a glass, round bottom tube followed by chloroform (200 $\mu 1)$ to dissolve lipid. Chloroform is evaporated in a stream of nitrogen with constant rotation to distribute the lipid evenly along the tube wall and placed under vacuum overnight. DMPC is either re-suspended in TBS with probe sonication or with TBS/cholate and gentle vortexing; the final concentration of cholate $(20 \mathrm{mM})$ is above its critical micellar concentration (CMC). Apolipoproteins (200-250 $\mu \mathrm{g})$ are added to the TBS/DMPC solution +/- cholate at a mass ratio of 4:1 for apoE422K and 3:1 of apoLpIII. The particle formation process is started with 3 repeated sets of transition temperature incubations, above and below the transition temperature of DMPC $\left(23.8^{\circ} \mathrm{C}\right)$, i.e. 10 minutes at $30^{\circ} \mathrm{C}$, then 10 minutes at $20^{\circ} \mathrm{C}$, with light hand mixing between incubations. After 3 heating and cooling transitions, the samples are incubated at $23.8^{\circ} \mathrm{C}$ overnight. 
Following assembly, samples containing cholate are dialyzed against $1000 \mathrm{x}$ volume of TBS buffer using 3 changes in 24 hrs. The NLPs are purified from 'free protein' and 'free lipid' by size-exclusion chromatography (VP HPLC, Shimadzu) using a Superdex 200 HR 10/30 column (GE Healthcare), in TBS at a flow rate of $0.5 \mathrm{ml} / \mathrm{min}$. The column was calibrated with four protein standards of known molecular weight and Stokes diameter that span the separation range of the column and the NLP samples. The void volume was established with Blue dextran. The NLP fractions are concentrated to approximately 0.1 $\mathrm{mg} / \mathrm{ml}$ using molecular weight sieve filters (Vivascience) with molecular weight cutoffs of $50 \mathrm{kDa}$. Protein concentration was determined using the ADV01 protein concentration kit (Cytoskeleton, Inc.).

SI Figure 1. Schematic of NLP assembly as described in Experimental Section. Individual reactants are combined, mixed and subjected to a series of temperature transitions before overnight incubation. NLPs are separated from the reaction mixture by chromatography, concentrated and characterized.

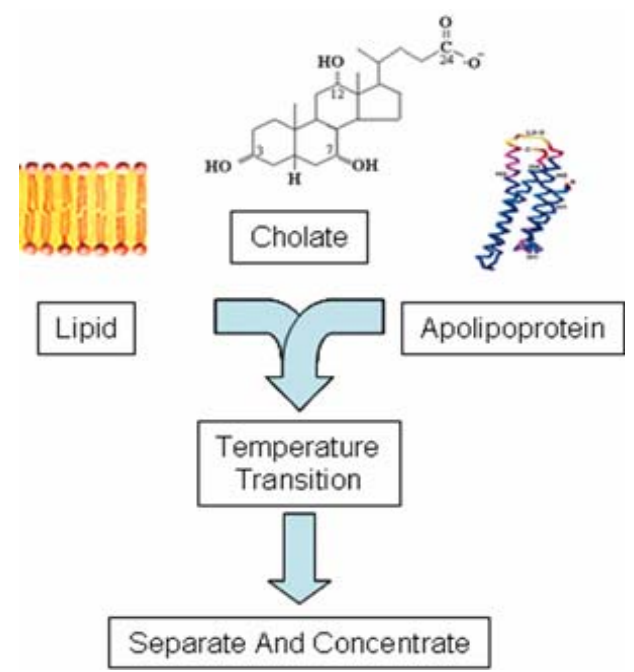

Native PAGE: Equal amounts of NLP samples $(0.5-2 \mu \mathrm{g})$ are diluted with $2 \mathrm{x}$ native gel sample buffer (Invitrogen) and loaded onto $4-20 \%$ gradient pre-made Tris- $\mathrm{HCl}$ gels (Invitrogen). Samples are electrophoresed for $250 \mathrm{Vhrs}$ at a constant $125 \mathrm{~V}$. After electrophoresis, gels are incubated with Sypro Ruby for 2 hours and then destained using $10 \% \mathrm{MeOH}, 7 \%$ Acetic acid. Following a brief wash with $\mathrm{ddH}_{2} \mathrm{O}$, gels are imaged using 
a Typhoon 9410 (GE Healthcare) at $532 \mathrm{~nm}$ (green laser) with a $610 \mathrm{~nm}$ bandpass 30 filter. Molecular weights are determined by comparing migration vs. log molecular weight of standard proteins found in the NativeMark standard (Invitrogen). The Stokes diameter of the NLPs is calculated from the known Stokes diameter of the same proteins in the standard sample.

Ion Mobility Spectrometry: The aerodynamic diameter of NLPs was determined with a Macroion Mobility Spectrometer (Model 3890, TSI Inc., Shoreview, MN). The details of the instrumentation and a method for measuring protein sizes have been described elsewhere ${ }^{4,5}$. Interestingly, this method has been used to measure the size distribution for HDL, LDL and VLDL taken directly from serum ${ }^{6}$. Briefly, the instrument consists of an electrospray ionization source with a charge-neutralizing chamber, a differential mobility analyzer (DMA) and a condensation particle counter (CPC). Multiply charged droplets generated by electrospray are charge-reduced by interaction with air ions formed by $\alpha$ radiation $\left({ }^{210} \mathrm{Po}\right)$. NLP samples are exchanged via dialysis (3x buffer exchange) into a volatile buffer and then pumped into the electrospray source at $100 \mathrm{~nL} / \mathrm{min}$. These conditions were chosen so primary electrospray droplets contain, on average, less than one individual NLP in $25 \mathrm{mM}$ ammonium acetate. The droplets ultimately evaporate, leaving individual NLPs in the gas phase carrying, predominantly, a single charge ${ }^{7}$. Charged NLPs pass through a scanning differential mobility analyzer and are counted by a condensation particle counter. The size distribution of a population of NLPs is determined from the scanning parameters; mobility measurements are used to infer NLP mean aerodynamic diameter. 
Mass Spectrometry: Mass determination was performed using Bruker APEX II 9.4 T FTICR mass spectrometer through a homebuilt nanospray interface on an Apollo (Bruker Daltonics, Billerica, MA, USA) ESI source. Protein solution concentrations were 1-10 $\mu \mathrm{M}$ or $1 \mathrm{nM}$ in $10 \mathrm{mM}$ ammonium acetate, $\mathrm{pH}$ 7.5. Solutions were desalted and concentrated by centrifugal filtration using Microcon or Amicon Ultra-4 filters (Millipore, Bedford, MA).

Atomic force microscopy (AFM): Atomically flat Muscovite mica disks were glued to metal substrates to secure them to the scanner of a stand-alone MFP-3D AFM (Asylum Research, Santa Barbara, CA). $2 \mathrm{uL}$ of solution was incubated for two minutes on the mica surface in imaging buffer $(10 \mathrm{mM} \mathrm{MgCl} 2,10 \mathrm{mM}$ Tris-HCL, and $0.1 \mathrm{M} \mathrm{NaCl}$, adjusted to $\mathrm{pH}$ 8.0) and then lightly rinsed. The AFM has a closed loop in the $\mathrm{x}, \mathrm{y}$, and $\mathrm{z}$ axes. The topographical images were obtained with "Biolevers" (Olympus, Tokyo, Japan) with a spring constant of $0.03 \mathrm{~N} / \mathrm{m}$. Images were taken in alternate contact (AC) mode in liquid, with amplitudes below $20 \mathrm{~nm}$ and an amplitude setpoint at 50\% tapping amplitude. Scan rates were below 1.5 Hz. Height, amplitude, and phase images were recorded. Heights of features in images were determined by histogram analysis of contiguous particles. Experiments were carried out in a temperature controlled room at 23 $+/-1{ }^{\circ} \mathrm{C}$. 
Transmission Electron Microscopy (TEM): Samples were diluted using TBS to achieve a final concentration of $0.02 \mathrm{mg} / \mathrm{ml}$. Three $\mu$ of each sample was pipetted onto a carbon coated 400 mesh copper EM grid (Ted Pella). After sitting for 1 minute, the sample was blotted with Whatman filter paper. Three $\mu$ of $2 \%$ Uranyl Acetate (Electron Microscopy Sciences) was applied for one minute and then blotted. Grids were dried for 30 minutes before use in the EM. Negative stain images were recorded using a Philips CM300 FEG transmission electron microscope operating at an accelerating voltage of $300 \mathrm{keV}$. Images were recorded as 8-bit and 16-bit tiff at varying magnifications onto a Gatan digital CCD and stored as jpegs and Gatan image format files. Images were then analyzed using Gatan Digital Micrograph software.

\begin{tabular}{|c|c|c|c|c|}
\hline Apolipoprotein & Mol. Wt. & Lipid & Cholate & Key REFs \\
\hline ApoAl & $28.1 \mathrm{kDa}$ & DMPC & No & Jonas et al., $1980^{8}$ \\
\hline MSP1T2 ( $\triangle 1-55$ ApoAl) & $24.8 \mathrm{kDa}$ & DMPC & No & $\begin{array}{l}\text { Sligar et al., } 2005^{9} \\
\text { Denisov et al., } 2005^{10}\end{array}$ \\
\hline ApoE422K & $22.3 \mathrm{kDa}$ & DMPC & No & Lu et al, $2000^{11}$ \\
\hline ApoLp-III & $18 \mathrm{kDa}$ & DMPC & No & $\begin{array}{l}\text { Weintzek, M. et al. } 1994^{12} ; \\
\text { Weers and Ryan, } 2003^{13}\end{array}$ \\
\hline ApoAl & $28.1 \mathrm{kDa}$ & DMPC & Yes & Jonas et al., $1980^{8}$ \\
\hline MSP1T2 ( $\triangle 1-55$ ApoAl) & $24.8 \mathrm{kDa}$ & DMPC & Yes & $\begin{array}{l}\text { Shaw et al., } 2004^{14} \\
\text { Bayburt et al., } 2006^{15}\end{array}$ \\
\hline ApoE422K & $22.3 \mathrm{kDa}$ & DMPC & Yes & This work \\
\hline ApoLp-III & 18 kDa & DMPC & Yes & Garda HA et al., $2002^{16}$ \\
\hline Су3-ароE422K & $22.3 \mathrm{kDa}$ & DMPC & Yes \& No & This work \\
\hline Су3-ароE422K & $22.3 \mathrm{kDa}$ & $\begin{array}{c}\text { DMPC } \\
+1 \% \\
\text { NBD- } \\
\text { DMPC }\end{array}$ & Yes \& No & This work \\
\hline
\end{tabular}

SI Table 1. Apolipoproteins: Four different apolipoproteins were chosen for this study. Each lipoprotein was studied for NLP formation with DMPC with and without cholate. Fluorescently labeled, Cy3-E422K was also used in this study to form NLPs, including in the presence of fluorescently labeled lipid (1\% NBD-DMPC). The molecular weight and key references for these apolipoprotein assemblies are shown. 
1. Morrow, J. A.; Arnold, K. S.; Weisgraber, K. H., Protein Expr Purif 1999, 16, (2), 224-30.

2. Ryan, R. O.; Schieve, D.; Wientzek, M.; Narayanaswami, V.; Oikawa, K.; Kay, C. M.; Agellon, L. B., J Lipid Res 1995, 36, (5), 1066-72.

3. Narayanaswami, V.; Yamauchi, Y.; Weers, P. M.; Maekawa, H.; Sato, R.;

Tsuchida, K.; Oikawa, K.; Kay, C. M.; Ryan, R. O., Eur J Biochem 2000, 267, (3), 728-

36.

4. Bacher, G.; Korner, R.; Atrih, A.; Foster, S. J.; Roepstorff, P.; Allmaier, G., J Mass Spectrom 2001, 36, (2), 124-39.

5. Loo, J. A.; Berhane, B.; Kaddis, C. S.; Wooding, K. M.; Xie, Y.; Kaufman, S. L.; Chernushevich, I. V., J Am Soc Mass Spectrom 2005, 16, (7), 998-1008.

6. Benner, W. H., R. Krauss and P. Blanche 20030136680, 2003.

7. Kaufman, S. L., Analytica Chimica Acta 2000, 406, 3-10.

8. Jonas, A.; Drengler, S. M.; Patterson, B. W., J Biol Chem 1980, 255, (5), 2183-9.

9. $\quad$ Sligar, S. G. B., T.H., Schuler, M.A., Civjan, N.R., Grinkova, Y.V., Denisov, I.G., and S.J. Grimme US 2005/0182243, Aug. 18, 2005, 2005.

10. Denisov, I. G.; McLean, M. A.; Shaw, A. W.; Grinkova, Y. V.; Sligar, S. G., J Phys Chem B Condens Matter Mater Surf Interfaces Biophys 2005, 109, (32), 15580-8.

11. Lu, B.; Morrow, J. A.; Weisgraber, K. H., J Biol Chem 2000, 275, (27), 20775-81.

12. Wientzek, M.; Kay, C. M.; Oikawa, K.; Ryan, R. O., J Biol Chem 1994, 269, (6), 4605-12.

13. Weers, P. M.; Ryan, R. O., Insect Biochem Mol Biol 2003, 33, (12), 1249-60.

14. Shaw, A. W.; McLean, M. A.; Sligar, S. G., FEBS Lett 2004, 556, (1-3), 260-4.

15. Bayburt, T. H.; Grinkova, Y. V.; Sligar, S. G., Arch Biochem Biophys 2006, 450, (2), 215-22.

16. Garda, H. A.; Arrese, E. L.; Soulages, J. L., J Biol Chem 2002, 277, (22), 1977382. 